\title{
Huntington-Krankheit und Chorea minor
}

Björn Falkenburger

Die Huntington-Krankheit war ein wichtiges Beispiel, an dem die Problematik prädiktiver genetischer Testung diskutiert wurde. Ebenso wie andere

Bewegungsstörungen schließt sie nichtmotorische Symptome und eine

Prodromalphase ein. Als rasch progrediente monogenetische Erkrankung ist sie eine wichtige Modellerkrankung zur Erforschung neurodegenerativer Pathomechanismen und eine der ersten Bewegungsstörungen, für die kausale Therapien greifbar nahe scheinen.

\begin{tabular}{|c|c|}
\hline \multicolumn{2}{|c|}{ ABKÜRZUNGEN } \\
\hline AFP & Alpha-Fetoprotein \\
\hline ANA & antinukleäre Antikörper \\
\hline ASO & Antisense-Oligonukleotid \\
\hline CAG & Cytosin, Adenin, Guanin \\
\hline CRP & C-reaktives Protein \\
\hline GABA & Gamma-Aminobuttersäure \\
\hline $\mathrm{HbA}_{1 \mathrm{c}}$ & Glykohämoglobin \\
\hline HCG & humanes Choriongonadotropin \\
\hline HIV & humanes Immundefizienzvirus \\
\hline MMSE & Mini Mental State Examination \\
\hline MoCA & Montreal Cognitive Assessment \\
\hline mRNA & $\begin{array}{l}\text { Messenger-RNA (ribonucleinic Acid; } \\
\text { Ribonukleinsäure) }\end{array}$ \\
\hline NBIA & $\begin{array}{l}\text { Neurodegeneration with Brain Iron } \\
\text { Accumulation }\end{array}$ \\
\hline NSAID & $\begin{array}{l}\text { Nonsteroidal anti-inflammatory } \\
\text { Drug (nichtsteroidales } \\
\text { Antiphlogistikum) }\end{array}$ \\
\hline PBA-s & $\begin{array}{l}\text { Problem-Behavior-Assessment (short } \\
\text { Version) }\end{array}$ \\
\hline PCR & $\begin{array}{l}\text { Polymerase Chain Reaction } \\
\text { (Polymerasekettenreaktion) }\end{array}$ \\
\hline PEG & perkutane endoskopische Gastrostomie \\
\hline PKAN & $\begin{array}{l}\text { Pantothenatkinase-assoziierte } \\
\text { Neurodegeneration }\end{array}$ \\
\hline SREAT & Steroid-responsive Enzephalopathie \\
\hline SSEP & somatosensibel evozierte Potenziale \\
\hline SSRI & $\begin{array}{l}\text { Selective Serotonin Reuptake Inhibitor } \\
\text { (selektiver Serotonin- } \\
\text { Wiederaufnahmehemmer) }\end{array}$ \\
\hline TEE & transösophageale Echokardiografie \\
\hline TSH & thyreoideastimulierendes Hormon \\
\hline UHDRS & $\begin{array}{l}\text { Unified Huntington's Disease Rating } \\
\text { Scale }\end{array}$ \\
\hline
\end{tabular}

\section{FALLBEISPIEL 1}

Die 66-jährige Patientin stellt sich vor, weil ihrem Sohn eine motorische Unruhe aufgefallen sei. Vor 8 Jahren war sie aufgrund einer als funktionell eingeschätzten Verdauungsstörung in Behandlung, seit 5 Jahren sei eine Angststörung bekannt. Depressive Symptome werden verneint. Manchmal vergesse sie Dinge oder Termine. Eine Einschränkung im Alltag bestehe nicht.

Klinisch zeigt sich ein unauffälliger Hirnnervenstatus, normotone Muskulatur der Extremitäten und keine Paresen, aber beidseitig eine geringe Verzögerung im Fingertippen. Es bestehen unwillkürliche Bewegungen im Bereich des Munds, an den Armen und Beinen, dort distal betont. Die Zeigeversuche sind dadurch etwas dysmetrisch, der Gang ist jedoch nicht relevant eingeschränkt, selbst der Seiltänzergang ist sicher. Die Patientin ist vollständig orientiert. Das Denken ist etwas umständlich, und von 3 Wörtern wird nur eines erinnert. Die Stimmung ist ausgeglichen, die Schwingungsfähigkeit erhalten. Die Patientin nimmt aktuell Paroxetin ein; die Einnahme von Neuroleptika in der Vergangenheit wird verneint. Ein cMRT ist unauffällig. Eine genetische Testung ergibt 40 CAG-Repeats im Huntington-Gen. Die daraufhin erweiterte Familienanamnese ergibt, dass der Vater der Patientin mit 75 Jahren in einem pflegebedürftigen, eher unterbeweglichen Zustand unklarer Ätiologie verstorben sei. Er habe schlecht schlucken und nicht mehr eigenständig gehen können. Die Mutter sei an einem kardiovaskulären Ereignis verstorben. Die Patientin habe keine Geschwister, aber 2 Kinder.

In der Zusammenschau diagnostizieren Sie eine Huntington-Krankheit. Sie klären die Patientin und ihren Sohn über die Huntington-Krankheit auf. Sie 
informieren den Sohn über die Tatsache, dass die Erkrankung vererbt werden kann und dass der Sohn sich nach einer genetischen Beratung auf die Erkrankung testen lassen kann. Er wünscht aktuell keine Beratung oder Testung. Sie überlegen, ob Sie eine Medikation gegen die Hyperkinesien beginnen sollen, entscheiden sich jedoch dagegen, da die Patientin sicher gehen kann. Sie führen die aktuelle Medikation mit Paroxetin fort und bieten eine Wiedervorstellung in 6 Monaten an.

\section{Huntington-Krankheit}

\section{Typische Präsentation}

Die Huntington-Krankheit ist eine autosomal-dominant vererbte Erkrankung mit hoher Penetranz. Viele Patienten kennen die Symptome daher vom erkrankten Elternteil und kommen direkt mit der Frage zum Neurologen, ob sie ebenfalls betroffen sind. Das Manifestationsalter liegt in der Regel zwischen dem 30. und 50. Lebensjahr. Es gibt daher Konstellationen, in denen die Familienanamnese leer erscheinen kann (Elternteil unklar, verstorben oder kein Kontakt zum Elternteil). Auch „Neumutationen“ sind möglich, insbesondere bei Patienten mit spätem Krankheitsbeginn (> 59. Lebensjahr) [1]).

\section{CAVE}

Eine offenbar negative Familienanamnese schließt die Huntington-Krankheit nicht aus.

\section{Epidemiologie und Verlauf}

Die Prävalenz der Huntington-Krankheit liegt bei ca. 5 pro 100000, das entspricht in etwa 8000-10000 Patienten in Deutschland. Die Inzidenz liegt bei 4:1 Million, entsprechend werden pro Jahr in Deutschland etwa 300 Patienten neu diagnostiziert.

Huntington-Patienten sterben im Mittel 15-20 Jahre nach Manifestation der ersten motorischen Symptome; es sind jedoch auch benigne Verläufe über 40 Jahre beschrieben. Todesursache ist häufig eine Pneumonie bei ausgeprägter Kachexie und Aspirationsneigung. Die Kachexie ist bedingt durch den erhöhten Energiebedarf aufgrund der Chorea, eine erschwerte Nahrungsaufnahme und metabolische Veränderungen. Auch Suizide sind häufig [2].

\section{Ätiologie und Pathogenese}

Die Huntington-Krankheit ist durch eine Expansion von CAG-Triplets im Huntington-Gen bedingt. Bei Gesunden enthält das Huntington-Gen 9-35 CAG-Wiederholungen, bei Patienten ist die Anzahl der Wiederholungen typischerweise auf über 40 erhöht. Mehr Wiederholungen sind dabei mit einem früheren Erkrankungsbeginn assoziiert.

Auch die Geschwindigkeit der motorischen Verschlechterung wird durch die Anzahl der Wiederholungen mitbestimmt [3]. Im Bereich zwischen 36 und 39 Wiederholungen ist die Penetranz der Erkrankung unvollständig. Trotz dieser Zusammenhänge kann bei einem einzelnen Patienten nicht von der Anzahl der CAG-Wiederholungen auf das Erkrankungsalter oder die Progressionsrate geschlossen werden.

In vielen Fällen nimmt die Anzahl der Wiederholungen von Generation zu Generation zu und das Manifestationsalter ab (Antizipation). Dieser Effekt ist besonders ausgeprägt, wenn das pathogene Allel vom Vater vererbt wird. Wenn durch die Antizipation die Anzahl der CAGWiederholungen aus dem normalen Bereich auf über 36 erhöht ist, kann die Erkrankung bei leerer Familienanamnese auftreten („Neumutation“).

Der verlängerte CAG-Abschnitt ist genetisch instabil, sodass sich die Länge der CAG-Wiederholungen nicht nur entlang der Keimbahn verändert, sondern auch innerhalb eines Körpers („somatische Instabilität“). Das Ausmaß dieser Instabilität unterscheidet sich zwischen verschiedenen Organen, Hirnregionen und Zelltypen. Diese Unterschiede können somit erklären, warum GABAerge Neurone im Striatum besonders früh degenerieren, obwohl das Huntington-Protein ubiquitär exprimiert wird.

\section{HINTERGRUNDWISSEN \\ Zellbiologie der Huntington-Krankheit}

Der dominante Erbgang der Huntington-Krankheit legt nahe, dass die Krankheit durch eine toxische Wirkung des mutierten Proteins entsteht. Die Expansion des CAG-Triplets führt nicht nur zu den bekannten Polyglutamin-Proteinen, sondern verändert auch die Sekundärstruktur der m-RNA (messenger-RNA). Die veränderte messenger-RNA kann die Transkription verändern und RNA-bindende Proteine im Zellkern „einfangen“. Darüber hinaus finden sich im Zellkern Ablagerungen fehlgefalteter Proteine, die nicht nur aus Polyglutamin-Anteilen bestehen, sondern auch aus Proteinen, die aus den CAG-Wiederholungen bei verschobenem Leseraster entstehen. Diese Proteinaggregate behindern den axonalen Transport und können den Zelltod auslösen.

Die Ausbildung der Einschlusskörper im Zellkern ist eine protektive Reaktion auf die fehlgefalteten Polyglutamin-Proteine [4]. Polyglutamin-Proteine können durch Autophagie abgebaut werden und durch 
unkonventionelle Exozytose aus der Zelle freigesetzt werden [5].

Darüber hinaus wird diskutiert, ob durch die verlängerten CAG-Wiederholungen physiologische Funktionen des Huntington-Proteins beeinträchtigt werden.

\section{Juvenile Variante}

Von der typischen Huntington-Krankheit wird eine juvenile Variante abgegrenzt, die etwa $5 \%$ der HuntingtonPatienten ausmacht. Viele dieser Patienten zeigen besonders lange CAG-Wiederholungen (> 60) und dementsprechend eine rasche Progredienz. Motorisch stehen in der juvenilen Variante hypokinetisch-rigide und dystone Symptome im Vordergrund, ähnlich wie es auch in der fortgeschrittenen typischen Huntington-Krankheit der Fall ist. Auch epileptische Anfälle können in dieser Verlaufsform auftreten.

\section{CAVE}

Bei der Huntington-Krankheit können nicht nur Hyperkinesien, sondern auch akinetisch-rigide Symptome auftreten.

\section{Diagnosestellung}

\section{Anamnese}

Wichtige Fragen in der Anamnese betreffen Verhaltensauffälligkeiten, psychiatrische Symptome (s. u. ) und eine genaue Familienanamnese. Zur differenzialdiagnostischen Abgrenzung gegenüber tardiven Dyskinesien sollte nach der Einnahme von Dopamin-Antagonisten gefragt werden-konkret nach Neuroleptika, „Aufbauspritzen" und Metoclopramid. Auch eine Schwangerschaft oder hormonelle Kontrazeptiva können in seltenen Fällen eine symptomatische Chorea auslösen, des Weiteren internistische Erkrankungen (systemischer Lupus erythematodes, Hyperthyreose, Tumorerkrankungen) und metabolische Veränderungen (Hyperglykämie, Elektrolytverschiebungen).

\section{Neurologischer Untersuchungsbefund - Chorea}

Oft ist die Beobachtung der Spontanbewegungen eines Patienten ausreichend, um den Verdacht auf eine Huntington-Krankheit zu lenken. Die klassische Bewegungsstörung der Huntington-Krankheit, die Chorea, besteht in einer Vermehrung kurzer, unwillkürlicher Bewegungen. Diese Bewegungen sind nicht stereotyp und können in allen Bereichen des Körpers auftreten. Jede für sich genommen kann zu Beginn der Erkrankung nicht sicher als abnorm eingeordnet werden, insgesamt sind es jedoch zu viele Bewegungen. Ähnlich wie Tics können choreatische Bewegungen in Willkürbewegungen auslaufen und dadurch „verborgen“ werden. Im Gegensatz zu Tics können choreatische Bewegungen jedoch nur zum Teil unterdrückt werden. Die choreatischen Bewegungen sind in der Regel symmetrisch ausgeprägt. Wie die meisten Bewegungsstörungen nimmt die Chorea bei Anspannung zu und sistiert im Schlaf. Ein typisches Zeichen für die Huntington-Krankheit ist die motorische Impersistenz. Dabei wird der Patient gebeten, die Zunge aus dem Mund herausstrecken und dort 5 oder 10 Sekunden zu halten. Patienten mit einer Huntington-Krankheit können die Zunge entweder gar nicht herausstrecken oder nur kurz dort halten.

\footnotetext{
Merke

Wie bei der Huntington-Krankheit besteht auch beim Tourette-Syndrom eine Kombination aus Hyperkinesen und psychiatrischen Symptomen. Tics sind jedoch stereotyper als choreatische Bewegungen und können zumindest kurzzeitig unterdrückt werden.
}

Falls sich der Verdacht auf eine Huntington-Krankheit erhärtet, ist ein Assessment nach der UHDRS (Unified Huntington's Disease Rating Scale) sinnvoll [15]. Sie erlaubt ein systematisches Assessment, welche Teile des motorischen Systems betroffen sind. Dabei werden beurteilt:

- langsame Augenfolgebewegungen,

- Sakkaden,

- Dysarthrie,

- Dystonie,

- Chorea,

- posturale Stabilität,

- langsames Fingertippen,

- Diadochokinese,

- Rigidität,

- Bradykinese,

- Gang.

Viele der Symptome sind nicht spezifisch für die Huntington-Krankheit. Ihre Quantifizierung erlaubt jedoch einen Vergleich mit Voruntersuchungen und damit eine Aussage über die Progredienz der Erkrankung.

\footnotetext{
Merke

Im Laufe der Erkrankungen werden die Bewegungen häufiger und ihre Amplitude nimmt zu, bevor die Chorea im Spätstadium gewissermaßen „ausbrennen“ kann.
}

Gehen, Sprechen und Essen werden durch die Chorea eingeschränkt und sind in fortgeschrittenen Stadien nicht mehr möglich. Der Muskeltonus ist zu Beginn der Erkrankung normal, im Verlauf dyston erhöht. Die Bewegungen können im fortgeschrittenen Stadium deutlich verlangsamt wirken und eher einer Parkinson-Symptomatik ähneln. 


\section{Differenzialdiagnosen}

Bei Erstdiagnose wird nach Feststellen und Quantifizierung der Chorea nach Hinweisen auf eine alternative Diagnose gesucht (s. Tab. 1). Untypisch wäre z. B. eine stark asymmetrische Ausprägung der Chorea oder eine Beschränkung auf das Gesicht. In diesen Fällen wäre(n) eine strukturelle Läsion im Bereich der Basalganglien bzw. tardive Dyskinesien auszuschließen.

Weitere differenzialdiagnostisch zu erwägende Erkrankungsgruppen sind die Neuroakanthozytose-Syndrome, zu denen die Chorea-Akanthozytose und das McLeodSyndrom zählen, und die Erkrankungen mit Neurodegeneration und Eisenablagerungen im Gehirn (NBIA, Neurodegeneration with Brain Iron Accumulation), zu denen die Pantothenatkinase-assoziierte Neurodegeneration (PKAN) und die Neuroferritinopathie zählen. Auch bei zerebellären Ataxien (spinozerebelläre Ataxien, Friedreich-Ataxie, Ataxia teleangiectatica, Ataxie mit okulomotorischer Apraxie) können Hyperkinesen auftreten. Zudem kann eine ausgeprägte Ataxie als Chorea fehlgedeutet werden.

\section{THERAPIE}

Behandelbare Differenzialdiagnosen

- Chorea minor

- Morbus Wilson

- Paraneoplasie

\section{Psychiatrischer Untersuchungsbefund}

Oft bestehen bereits vor Manifestation der motorischen Symptome Verhaltensauffälligkeiten. Die Patienten sind oft sozial unangepasst oder schlecht integriert. Typisch sind zudem Reizbarkeit oder gewalttätiges Verhalten mit Drohungen und Wutausbrüchen.

Da sich viele dieser Symptome nur eingeschränkt objektiv beurteilen lassen, hat es sich bewährt, nach dem Ausmaß des „sozialen Funktionierens“ zu fragen: Ist der Patient berufstätig? Kann er seine finanziellen Geschäfte regeln? Kann er Arbeiten im Haushalt verrichten? etc. Strukturiert lassen sich diese Aspekte mit den Teilen „Functional Assessment“ und „Functional Capacity“ des UHDRS erfassen (s. Infobox).

\Tab. 1 Symptome, die an der Diagnose Huntington-Krankheit zweifeln lassen.

\begin{tabular}{|c|c|}
\hline Symptom & Differenzialdiagnose \\
\hline einseitige und proximal betonte Hyperkinesien & Hemiballismus (z. B. nach Schlaganfall im Nucleus subthalamicus) \\
\hline vornehmlich orale und linguale Hyperkinesien & $\begin{array}{l}\text { tardive Dyskinesien Neuroakanthozytose NBIA (Neurodegenera- } \\
\text { tion with Brain Iron Accumulation) }\end{array}$ \\
\hline Pharyngitis & Chorea minor \\
\hline anfallsartige Chorea für Minuten bis Stunden & paroxysmale kinesiogene Dyskinesie \\
\hline plötzlicher Beginn der Chorea & vaskuläre Genese Chorea minor \\
\hline ausgeprägte Selbstverletzungen & Lesch-Nyhan-Syndrom \\
\hline Teleangiektasien der Sklera & Ataxia teleangiectatica \\
\hline Kayser-Fleischer-Kornealring & Morbus Wilson \\
\hline Retinitis pigmentosa & NBIA (Neurodegeneration with Brain Iron Accumulation) \\
\hline ausgeprägte zerebelläre Okulomotorikstörung & spinozerebelläre Ataxie Friedreich-Ataxie \\
\hline Zeichen einer Motoneuronerkrankung & C9ORF72-Mutation \\
\hline Polyneuropathie & $\begin{array}{l}\text { spinozerebelläre Ataxie Friedreich-Ataxie Ataxiemit okulomotori- } \\
\text { scher ApraxieNeuroakanthozytose }\end{array}$ \\
\hline epileptische Anfälle & Neuroakanthozytose juvenile Huntington-Krankheit \\
\hline rasch progrediente Demenz, Myoklonien & Creutzfeldt-Jakob-Erkrankung \\
\hline Fehlen psychiatrischer Symptome & benigne familiäre Chorea \\
\hline Lebervergrößerung & Morbus Wilson Neuroakanthozytose \\
\hline Kardiomyopathie & Friedreich-Ataxie Neuroakanthozytose \\
\hline Endokarditis & Chorea minor \\
\hline Fieber & Chorea minor \\
\hline Gewichtsverlust, B-Symptomatik, negative Genetik & paraneoplastische Ursache \\
\hline
\end{tabular}




\section{ÜBERSICHT}

\section{Functional Capacity}

Mit den folgenden Fragen kann eingeschätzt werden, inwiefern der Patient durch die Erkrankung eingeschränkt ist. Die Fragen sind der Einfachheit halber für einen männlichen Patienten formuliert.

\section{Beruf}

- Kann der Patient in seinem Beruf arbeiten?

- Geht der Patient einer bezahlten Tätigkeit nach?

- Geht der Patient einer ehrenamtlichen oder unbezahlten Tätigkeit nach?

\section{Finanzen}

- Kann der Patient seine finanziellen Angelegenheiten ohne Hilfe regeln?

- Kann der Patient ohne Hilfe einkaufen gehen?

- Kann der Patient ohne Hilfe im Geschäft bezahlen?

\section{Verkehr}

- Kann der Patient sicher Auto fahren?

- Kann der Patient ohne Hilfe öffentliche Verkehrsmittel benutzen?

- Kann der Patient ohne Hilfe Orte in der Nachbarschaft aufsuchen?

\section{Haushalt}

- Kann der Patient seinen Haushalt ohne Hilfe führen?

- Kann der Patient ohne Hilfe seine Wäsche waschen?

- Kann der Patient ohne Hilfe eine Mahlzeit zubereiten?

\section{Körperpflege}

- Kann der Patient ohne Hilfe essen?

- Kann sich der Patient ohne Hilfe anziehen?

- Kann sich der Patient ohne Hilfe waschen/ duschen?

- Kann sich der Patient ohne Hilfe die Haare kämmen?

- Kann der Patient ohne Hilfe die Toilette benutzen?

\section{Mobilität}

- Stürzt der Patient häufig?

- Kann der Patient ohne Hilfe gehen?

- Kann der Patient ohne Hilfe aus dem Stuhl aufstehen?

- Kann der Patient ohne Hilfe aus dem Bett aufstehen?

Diese Fragen liefern wichtige Hinweise darauf, in welchen Bereichen der Patient eingeschränkt ist und ggf. Hilfe benötigt. Zur Verlaufsbeobachtung kann das Ausmaß der Beeinträchtigung wie folgt quantifiziert werden:

\section{Beruf}

- 0 - keine Tätigkeit möglich

- 1 - nur einfache Tätigkeiten

- 2 -reduziert im eigentlichen Beruf

- 3-normal

\section{Finanzen}

- 0 - unmöglich

- 1 - mit viel Hilfe

- 2 - mit geringer Hilfe

- 3-vollständig unabhängig

Haushalt

- 0 - unmöglich

- 1 -mit Hilfe

- 2 -vollständig unabhängig

Tägliches Leben

- 0 -vollständig pflegebedürftig

- 1 - nur einfache Aktivitäten

- 2 -geringe Einschränkungen

- 3 -vollständig unabhängig

\section{Ausmaß der Versorgung}

- 0 - institutionelle Pflege oder 24-StundenPflegekraft

- 1 -zu Hause, ggf. mit Pflegedienst

- 2 - unabhängig zu Hause

Viele Patienten leiden unter depressiven Symptomen. Daher sollten Stimmung (Neigung zum negativen Pol, Schwingungsfähigkeit) und Selbstwertgefühl erfasst werden und regelmäßig nach Suizidalität gefragt werden. Bei fortgeschrittener Krankheit kommen zunehmend auch Halluzinationen und inhaltliche Denkstörungen mit Wahninhalten vor. Strukturiert können diese Symptome z. B. mit der kurzen Version des Problem-Behavior-Assessment (PBA-s, s. [6]) erfragt werden.

\section{Kognitive Funktion}

Kognitive Einschränkungen gehören zur Erkrankung; typisch ist ein frontales/subkortikales Defizit. Die Luria-Sequenz ist daher häufig pathologisch. Weitere Frontalhirnsymptome sind die aus dem MoCA-Test bekannte Aufgabe, innerhalb einer Minute so viele Worte zu finden wie möglich, die mit dem Buchstaben „F“ beginnen, und Klatschaufgaben aus der „Frontal Assessment Battery“ wie „Klatschen Sie einmal, wenn ich zweimal klatsche, und klatschen Sie zweimal, wenn ich einmal klatsche“ oder „Klatschen Sie einmal, wenn ich einmal klatsche und klatschen Sie nicht, wenn ich zweimal klatsche“. Zur Quantifizierung geeignet ist darüber hinaus der Stroop-Test, bei dem z.B. das Wort "Grün“ in der Farbe Rot geschrieben ist, und wo entweder die Farbe benannt oder das Wort gelesen werden müssen.

Auch Aufmerksamkeit und Gedächtnis sind eingeschränkt. In der klinischen Routine wird als ScreeningTest meist der Montreal Cognitive Assessment (MoCA) verwendet, da er im Vergleich zum MMSE Störungen der Exekutivfunktionen besser erfasst und einen breiteren Bereich an Veränderungen abdeckt. 
Statistisch gesehen ist eine Reihe von kognitiven Tests lange (zum Teil mehr als 15 Jahre) vor Beginn der motorischen Symptome auffällig. Eine Beurteilung beim einzelnen Patienten ist dagegen oft schwierig, da individuelle Faktoren wie Schulbildung und Ausgangsintelligenz berücksichtigt werden müssen.

\section{HINTERGRUNDWISSEN}

Zu den frühen Auffälligkeiten gehören die Schwierigkeit, Emotionen anhand von Gesichtsausdrücken zu erkennen, sowie Schwierigkeiten im Abschätzen von Zeitintervallen.

\section{Zusatzdiagnostik}

Charakteristisches Zeichen der Huntington-Krankheit im zerebralen MRT ist eine Atrophie des Nucleus caudatus, die in koronaren Schnitten besonders deutlich ist. Das Ausmaß dieser Atrophie kann als Verlaufsparameter herangezogen werden [7]. Im Verlauf der Erkrankung zeigt sich darüber hinaus eine globale Hirnvolumenminderung.

\section{Merke}

Typisches MRT-Zeichen der Huntington-Krankheit: Atrophie des Nucleus caudatus.

Zur differenzialdiagnostischen Abgrenzung relevant sind der Ausschluss verschiedenster Läsionen (Infarkte,
Tumoren) im Striatum. Eisenablagerungen („Eye-of-theTiger-Zeichen“) sind typisch für NBIA. Auch ein Morbus Fahr mit Verkalkungen im Striatum kann in seltenen Fällen mit einer Chorea einhergehen. Typisch für eine Chorea bei nicht ketotischer Hyperglykämie ist eine flaue T1Hyperintensität im Striatum. Chorea verursachende Läsionen sind jedoch nicht auf das Striatum beschränkt. So wird eine proximal betonte Hemichorea (Hemiballismus) typischerweise durch eine Läsion im Nucleus subthalamicus verursacht. Relevant ist schließlich der Ausschluss einer Atrophie des Zerebellums als Zeichen einer spinozerebellären Ataxie.

Elektrophysiologische Charakteristika (Riesen-SEP und Long-Latency-Reflexe) spielen seit Verfügbarkeit der genetischen Testung nur noch eine untergeordnete Rolle. Sinnvolle Laborwerte zur differenzialdiagnostischen Abgrenzung fasst $\triangleright$ Tab. 2 zusammen.

\section{Merke}

Die Diagnose Huntington-Krankheit wird in der Regel durch eine genetische Testung bestätigt.

Bei klinisch betroffenen Patienten kann die Abnahme von Blut zur genetischen Testung-nach Aufklärungdurch den betreuenden Arzt erfolgen. Das Untersuchungsergebnis muss dann persönlich und nach erneuter Aufklärung mitgeteilt werden. Bei positiven Befunden muss eine genetische Beratung angeboten werden. Die

-Tab. 2 Labordiagnostik zum Ausschluss relevanter Differenzialdiagnosen.

\begin{tabular}{|c|c|c|}
\hline Material & Wert & Differenzialdiagnose \\
\hline \multirow[t]{11}{*}{ Blut-Routinediagnostik } & $\mathrm{HbA}_{1 \mathrm{c}} \uparrow$ & Hypo- oder hyperglykäme Chorea \\
\hline & Alpha-Fetoprotein (AFP) $\uparrow$ & $\begin{array}{l}\text { Ataxia teleangiectatica (Ataxie mit okulomo- } \\
\text { torischer Apraxie) }\end{array}$ \\
\hline & Albumin $\downarrow$, Cholesterol $\uparrow$ & Ataxie mit okulomotorischer Apraxie \\
\hline & $\beta$-HCG $\uparrow$ & Schwangerschafts-Chorea \\
\hline & Harnsäure $\uparrow$ & Lesch-Nyhan-Syndrom \\
\hline & Leberenzyme $\uparrow$ & Morbus Wilson Neuroakanthozytose \\
\hline & Ferritin $\downarrow$ & $\begin{array}{l}\text { Neurodegeneration with Brain Iron Accumula- } \\
\text { tion (NBIA) }\end{array}$ \\
\hline & $\mathrm{TSH} \uparrow$ & Steroid-responsive Enzephalopathie (SREAT) \\
\hline & Coeruloplasmin $\downarrow$ & Morbus Wilson \\
\hline & $\begin{array}{l}\text { antinukleäre Antikörper Phospholipid- } \\
\text { Antikörper }\end{array}$ & systemischer Lupus erythematodes \\
\hline & HIV-Test & HIV-Enzephalopathie \\
\hline \multirow[t]{2}{*}{ Liquor } & Zellzahl $\uparrow$ oligoklonale Banden & Enzephalitiden \\
\hline & T. whipplei PCR & Morbus Whipple \\
\hline \multirow[t]{3}{*}{ Blut-erweiterte Diagnostik } & Akanthozyten im Blutausstrich & Neuroakanthozytose (PKAN) \\
\hline & Anti-Streptolysin O Anti-DNAse B & Chorea minor \\
\hline & neuronale Antikörper & paraneoplastische Genese \\
\hline
\end{tabular}


genetische Testung nicht betroffener Familienangehöriger muss dagegen immer durch einen Arzt erfolgen, der nach $\S 7$ GenDG qualifiziert ist. Eine psychologische Betreuung und die Richtlinien der „International Huntington Association“ können hierbei hilfreich sein. Bei positiver Familienanamnese und typischer Symptomatik kann ggf. auf die genetische Diagnosesicherung verzichtet werden.

\section{Merke}

Die genetische Testung betroffener Patienten kann durch den behandelnden Arzt eingeleitet werden. Eine genetische Beratung ist dennoch sinnvoll. Bei nicht betroffenen Familienmitgliedern muss die Testung dagegen durch einen Humangenetiker erfolgen.

Falls die genannte Diagnostik unergiebig bleibt, sind die Bestimmung neuronaler Antikörper und eine Tumorsuche (PET-CT) sinnvoll, um eine seltene paraneoplastische Genese der Symptomatik auszuschließen.

\section{FALLBEISPIEL 2}

Ein 65-jähriger Patient stellt sich in Begleitung seiner Ehefrau zur Verlaufskontrolle bei bekannter Huntington-Krankheit vor. Überbeweglichkeiten treten nur selten auf und beeinträchtigen den Patienten nicht im Alltag. Der Patient könne noch einige Kilometer frei gehen, Stürze sind bisher nicht zu verzeichnen. Die Stimmung sei meist ausgeglichen; immer wieder sei er jedoch schneller gereizt als früher. Der Antrieb sei reduziert, er gehe aus eigener Initiative nicht mehr seinen Hobbys nach. Das Gedächtnis sei eingeschränkt, die Orientierung in einer neuen Umgebung falle ihm schwer. Der Appetit sei gut, er esse manchmal etwas überstürzt, verschlucke sich jedoch nicht.

An Medikamenten nimmt der Patient $3 \times 25 \mathrm{mg}$ Nitoman ein.

Klinisch ist der Patient zu Person, Ort und Zeit orientiert. Die Stimmung ist ausgeglichen, die Schwingungsfähigkeit erhalten. Suizidale Gedanken werden glaubhaft verneint. Der Gedankengang ist umständlich und z. T. eingeengt. Wahrnehmungsstörungen oder inhaltliche Denkstörungen bestehen nicht.

Die Blickfolge ist sakkadiert, das Sprechen ist etwas verwaschen, aber noch gut verständlich, die Zunge kann herausgestreckt, aber nur für 6 Sekunden dort gehalten werden. An den Händen findet sich eine geringgradige Dystonie, Chorea ist lediglich intermittierend nachweisbar. Das Fingertippen ist rechts mäßig und links gering verlangsamt, PronationsSupinations-Bewegungen ebenfalls. Die LuriaSequenz kann nicht durchgeführt werden. Ein Rigor findet sich nicht, der Gang ist breitbasig. Im Zugtest fängt sich der Patient selbst auf.
Aufgrund der im Vordergrund stehenden depressiven Störung und der guten Kontrolle der Hyperkinesien empfehlen Sie eine schrittweise Reduktion der Medikation mit Nitoman auf $3 \times 12,5 \mathrm{mg}$. Sie erwarten davon außerdem eine Besserung der hypokinetischen Symptome. Sie verordnen wöchentliche Physiotherapie und Ergotherapie. Aufgrund von Reizbarkeit und Antriebsstörung erwägen Sie eine Medikation mit einem SSRI, beschließen jedoch, zunächst den Effekt der Nitoman-Reduktion abzuwarten.

\section{Therapie}

\section{Motorische Symptome}

Zur Reduktion der Chorea werden meist Tiaprid, Tetrabenazin oder eine Kombination aus beiden Medikamenten eingesetzt. Tetrabenazin ist hierbei oft wirksamer, kann jedoch eine Depression auslösen oder verschlechtern. Da Huntington-Patienten häufig ohnehin unter depressiven Symptomen leiden, wird die Therapie meist mit Tiaprid begonnen und bei nicht ausreichender Wirksamkeit auf Tetrabenazin umgestellt oder um Tetrabenazin ergänzt.

- Tiaprid wird um $50 \mathrm{mg}$ alle 2-4 Wochen gesteigert, eine Dosis von $4 \times 300 \mathrm{mg}$ pro Tag wird nur selten überschritten.

- Tetrabenazin wird um 12,5 mg alle 2-4 Wochen gesteigert bis maximal $4 \times 50 \mathrm{mg}$ pro Tag.

\section{THERAPIE}

\section{Medikamentöse Therapie}

- Tiaprid - besseres Nebenwirkungsprofil.

- Tetrabenazin - effektiver gegen Chorea.

Auch andere Dopamin-Antagonisten können eingesetzt werden. Aufgrund ihrer ko-antidepressiven Wirkung sind Quetiapin und Aripiprazol eventuell besonders geeignet. In der Praxis werden auch Risperidon, Olanzapin und ggf. Zuclopenthixol eingesetzt. Zu erwähnen ist, dass für keine der genannten Substanzen randomisierte klinische Studien mit hohen Fallzahlen vorliegen und die Empfehlungen daher im Wesentlichen auf Expertenmeinungen und kleinen Fallserien beruhen.

\section{TIPPS}

- Alle genannten Medikamente können die Beweglichkeit durch Verstärkung der akinetisch-rigiden Komponente der Huntington-Krankheit einschränken. Daher sollten choreatische Bewegungen nur dann medikamentös behandelt werden, wenn sie den Patienten funktionell relevant einschränken. 
- Zudem sollte die Medikation langsam gesteigert werden, um auf ggf. verzögert auftretende Verschlechterungen reagieren zu können.

- Da es sich um eine chronische Erkrankung handelt, ist eine gute Verträglichkeit wichtiger als ein rascher Wirkungsbeginn.

- Da die Chorea im Verlauf der Erkrankung oft abnimmt und die akinetisch-rigide Komponente an Bedeutung gewinnt, ist bei guter Kontrolle der Chorea immer wieder eine Dosisreduktion zu erwägen.

Falls sich die Chorea rasch verschlechtert, sollte eine interkurrierende Erkrankung ausgeschlossen werden, insbesondere ein Infekt oder bei häufigen Stürzen ein Subduralhämatom.

Bei ausgeprägten akinetisch-rigiden Symptomen sollte die Dosis der gegen die Chorea eingesetzten Medikamente verringert werden. Zudem kann eine Umstellung auf Substanzen mit einem geringeren Risiko für extrapyramidalmotorische Nebenwirkungen versucht werden (eher Tiaprid als Tetrabenazin und eher Quetiapin und Aripiprazol als Olanzapin, Risperidon oder Zuclopenthixol); die Wirkung von Clozapin auf choreatische Symptome ist dagegen unbefriedigend.

Dystone Symptome sind schwer zu behandeln. Möglicherweise können Cannabinoide eingesetzt werden [8], [9].

Falls der Patient durch starken Speichelfluss relevant beeinträchtigt ist, kann ein Versuch mit einer abendlichen Einnahme von Amitriptylin (trizyklisches Antidepressivum mit anticholinerger Wirkung, 25-50 mg) unternommen werden. Alternativ sind oral appliziertes Atropin oder die Injektion von Botulinum-Toxin in die Speicheldrüsen denkbar. Der Einsatz von Scopolamin-Pflastern oder anderer systemisch anticholinerg wirkender Substanzen ist zur Symptomkontrolle möglich, in vielen Fällen jedoch aufgrund der negativen Auswirkungen auf die ohnehin beeinträchtigte Kognition problematisch.

\section{CAVE}

Anticholinerg wirkende Substanzen können Speichelfluss, Schlafstörung, Unruhe und Depression behandeln, aber kognitive Defizite verstärken.

\section{Psychiatrische Symptome}

Wie oben ausgeführt finden sich bei der HuntingtonKrankheit eine Vielzahl von psychiatrischen Symptomen. Im Prinzip unterscheidet sich die Behandlung dieser
Symptome nicht von der Behandlung anderer Patienten. Man sollte jedoch vermeiden, für jedes Symptom ein eigenes Medikament zu verordnen. Vielmehr sollte man die Medikamente so auswählen, dass jeweils mehrere Symptome behandelt und negative Auswirkungen auf andere Symptome minimiert werden. Einem neuen Symptom würde man dementsprechend mit einer Dosisänderung oder mit dem Austauschen eines Medikaments begegnen.

Suizidgedanken kommen in allen Erkrankungsstadien vor und sind mit Angst und Depression assoziiert [2]. Deren suffiziente Behandlung senkt daher Morbidität und Mortalität der Huntington-Krankheit. Depressive Symptome werden im Wesentlichen so behandelt wie bei anderen Patienten mit Depression. Gute Erfahrungen bestehen mit Venlafaxin, das auch bei Angststörungen helfen kann. Sulpirid, Quetiapin und Aripiprazol können zur Augmentation eingesetzt werden und als Dopamin-Antagonisten ggf. auch die Chorea positiv beeinflussen.

Gegen Reizbarkeit und Stimmungsschwankungen können SSRI, Clonazepam und Valproat eingesetzt werden. Clonazepam kann darüber hinaus anxiolytisch, antidepressiv und antipsychotisch wirken. Die Wirkung ist durch einen Gewöhnungseffekt limitiert; das Suchtpotenzial ist aufgrund der progredienten Erkrankung meist weniger relevant. Auch Opipramol kann ängstliche Symptome und „innere Unruhe“ bessern. Neuere Daten legen darüber hinaus nahe, dass Reizbarkeit durch Cannabinoide (Sativex oder Dronabinol) reduziert werden kann [8], [9].

Psychotische Symptome wie Halluzinationen und Wahn können in frühen Erkrankungsstadien mit vorherrschender Chorea durch die dort genannten Neuroleptika behandelt werden (Quetiapin, Aripiprazol, Risperidon, Olanzapin). Schwere Psychosen im fortgeschrittenen Stadium mit vorherrschender akinetisch-rigider Symptomatik sollten dagegen mit Clozapin behandelt werden. Hierbei sind die vorgeschriebenen Blutbildkontrollen zu beachten (Agranulozytoserisiko).

Bei Schlafstörungen können durch Mirtazapin oder Amitriptylin eingesetzt werden. Leider kann durch Acetylcholinesterase-Hemmer keine Verbesserung der Kognition erreicht werden.

\section{Nichtmedikamentöse Therapien}

Eine Verbesserung des Gehens durch intensive Physiotherapie ist belegt. Zur Orientierung wurde durch das europäische Huntington-Netzwerk eine entsprechende Leitlinie entwickelt [11]. Darüber hinaus sind Ergotherapie und Logopädie (s. u.) sinnvoll. Eine stationäre Aufnahme zur intensivierten Therapie analog zur ParkinsonKomplexbehandlung wäre daher sinnvoll; die Behandlung wird allerdings aktuell nicht in der gleichen Form 
erstattet und erfordert aufgrund der psychiatrischen Symptome mehr pflegerische Ressourcen als die Behandlung von Parkinson-Patienten.

\section{Merke}

Nichtmedikamentöse Therapien spielen eine zentrale Rolle in der Versorgung von Huntington-Patienten [10].

Aufgrund der Verhaltensauffälligkeiten und der kognitiven Defizite sind sozialmedizinische Aspekte besonders wichtig, aber leider auch besonders schwierig. Die Behördengänge (für Arbeitsfähigkeit, Grad der Behinderung etc.) können im Verlauf häufig nicht mehr von den Patienten alleine bewältigt werden, sodass frühzeitig eine gesetzliche Betreuung eingerichtet werden sollte.

\section{TIPP}

Regelmäßig (ca. einmal pro Jahr) sollte die Frage diskutiert werden, wie am Lebensende verfahren werden soll. Konkret geklärt werden sollten die Fragen,

- ob zur Ernährung eine PEG-Sonde gelegt werden soll,

- ob zur Beatmung eine Tracheotomie erfolgen soll,

- ob eine Reanimation gewünscht ist oder abgelehnt wird.

Allen Patienten sollte eine psychosomatische Anbindung angeboten werden, um Strategien zur Krankheitsverarbeitung zu erarbeiten. An manchen Standorten können dazu Strukturen benutzt werden, die für onkologische Patienten etabliert wurden. Nach diesem Vorbild ist auch eine intensive Angehörigenarbeit sinnvoll.

Um die Kachexie zu verhindern, ist eine hyperkalorische Ernährung erforderlich. Falls aufgrund der Schluckstörung nicht ausreichend Kalorien aufgenommen werden können, sind hyperkalorische Drinks und eine PEG-Sonde indiziert. Eine logopädische Mitbehandlung ist sinnvoll, um Dysarthrie und Dysphagie zu verbessern und das Risiko für eine Aspiration zu kennen. Sobald eine relevante Schluckstörung besteht, kann das Andicken von Flüssigkeiten und das Passieren der Kost sinnvoll sein.

\section{Neuroprotektive Therapien}

Bislang gibt es keine neuroprotektive Therapie für die Huntington-Krankheit. Zahlreiche Therapieversuche waren nicht wirksam und sind daher obsolet, u. a. mit Coenzym Q, Vitamin A, C oder E, Kreatin, Riluzol, Idebenon, Remacemid oder Minocyclin. Auch eine symptomatische Therapie mit Pridopidin war nicht wirksam [12].

Aktuelle Studien zielen darauf ab, die genetische Ursache der Huntington-Krankheit zu behandeln. Dazu werden gegen das Huntington-Gen gerichtete Antisense-Oligonukleotide (ASO) eingesetzt. Zur besseren Bioverfügbarkeit wurden diese ASO chemisch modifiziert; dennoch müssen sie intrathekal verabreicht werden. Die verwendete Technologie ist damit ähnlich wie bei der spinalen Muskelatrophie. Mit diesen ASO konnte eine Reduktion des Huntington-Proteins im Liquor erreicht werden; ein Wirksamkeitsnachweis auf die klinischen Symptome wird in laufenden Studien untersucht.

Ein weiterer Ansatz besteht darin, die Manifestation der Erkrankung zu verzögern oder den Verlauf zu verlangsamen. Dazu wird nach Faktoren gesucht, die bei gleicher Anzahl von CAG-Wiederholungen das Manifestationsalter oder die Progredienz bestimmen. Ein verwandter Ansatz besteht darin, die somatische Instabilität zu vermindern, die für eine potenzierte Expansion der CAGWiederholungen im Gehirn und dort insbesondere im Striatum verantwortlich ist.

Eine tiefe Hirnstimulation mit Zielpunkt Globus pallidus konnte in wenigen Fällen motorische Symptome der Huntington-Krankheit bessern [13]. Eine Bestätigung in einem größeren Kollektiv steht allerdings noch aus. Bei zwei Patienten mit juveniler Huntington-Krankheit erbrachte eine tiefe Hirnstimulation im Globus pallidus keine Besserung [14]. In jedem Fall muss zwischen dem Nutzen für die motorischen Symptome und den von der Stimulation nicht veränderten kognitiven Einschränkungen abgewogen werden. Eine Behandlung sollte in klinischen Studien erfolgen.

TIPP: WEB-RESSOURCEN

https://huntington-hilfe.de

http://www.ehdn.org

https://chdifoundation.org

https://de.hdbuzz.net

\section{Chorea minor}

Die Chorea minor tritt meist bei Kindern auf, kann aber auch bei Erwachsenen vorkommen; Frauen sind häufiger betroffen als Männer.

\section{Klinik, Diagnostik}

Im Gegensatz zur langsam progredienten HuntingtonKrankheit ist der Beginn subakut. Die Bewegungsstörung an sich ist vergleichbar mit der Huntington-Krankheit; eine einseitige Ausprägung ist jedoch möglich. Die Bewegungsstörung kann wie die Huntington-Krankheit von psychiatrischen Symptomen und kognitiven Einschränkungen begleitet sein. Häufig sind Reizbarkeit, Stimmungsschwankungen und Aufmerksamkeitsstörung, aber auch Zwänge und Halluzinationen kommen vor. 
Die Krankheit ist eine Autoimmunenzephalitis und tritt meist Wochen bis Monate nach einer Infektion mit Streptokokken auf. Ein positiver Titer für Anti-Streptolysin O oder Anti-DNAse B kann die Diagnose stützen; ein negativer Titer schließt die Erkrankung jedoch nicht aus. Die MRT kann Signalveränderungen in Striatum oder Kortex zeigen. Beweisend für die Diagnose ist neben dem klinischen Bild insbesondere der benigne Verlauf: Die Bewegungsstörung selbst lässt in der Regel spontan innerhalb von Wochen bis Monaten nach. Symptomatisch kann sie zudem mit Tiaprid, Valproat oder Benzodiazepinen (z. B. Clonazepam) behandelt werden.

\section{CAVE}

Eine unerkannt persistierende Infektion, insbesondere eine Endokarditis, ist die wichtigste Ursache für einen ungünstigen Verlauf der Chorea minor.

\section{FALLBEISPIEL 3}

Eine 53-jährige Patientin wird aufgrund einer seit 2 Tagen bestehenden Bewegungsstörung in die Notaufnahme gebracht. Sie zeigt klinisch unwillkürliche, schraubenförmige Bewegungen der Hände (links mehr als rechts) und des linken Beins. Sie gehen aufgrund des akuten Auftretens zunächst von einer zerebralen Ischämie aus. Ein CCT und in der Folge ein CMRT sind jedoch unauffällig. Es zeigen sich keine ischämischen Läsionen und keine Eisenablagerungen im Striatum. Laborchemisch finden sich keine Hinweise auf metabolische Veränderungen, insbesondere normale Werte für Blutzucker, $\mathrm{HbA}_{1 \mathrm{c}}$, Elektrolyte, Leber- und Nierenwerte, Schilddrüsenwerte und Schilddrüsen-Antikörper. Das CRP ist leicht erhöht, Rheumafaktor, ANA und Phospholipid-Antikörper sind unauffällig. Im Blutausstrich finden sich keine Akanthozyten. Im Liquor werden normale Werte für Zellzahl und Eiweiß bestätigt, oligoklonale Banden stehen noch aus. Da Sie keine Erklärung finden, verschicken Sie Serum zur Bestimmung neuronaler Antikörper.

Am Folgetag berichtet die Tochter der Patientin von einer Pneumonie 3 Wochen vor der aktuellen Aufnahme. Sie bestimmen daraufhin Antikörper gegen Streptolysin O und DNAse B, die positiv sind. Sie gehen in der Summe von einer Chorea minor aus und beginnen eine Medikation mit Penicillin G $(3 \times$ 3 Mio. IE i. v. über 10 Tage).

Wiederholte Blutkulturen bleiben steril, ein TEE zeigt keinen Hinweis auf eine Endokarditis, eine Abdomensonografie findet ebenfalls keinen Fokus, es gibt keinen Hinweis auf eine Arthritis.

Die Patientin leidet subjektiv unter der Bewegungsstörung; sie berichtet von Schmerzen im Bereich der vermehrt belasteten Gelenke und sagt, sie traue sich mit den Symptomen nicht in Gesellschaft.
Sie beginnen eine Medikation mit Tiaprid und steigern um $50 \mathrm{mg}$ pro Tag bis $3 \times 100 \mathrm{mg}$. Um die Rückbildung der Bewegungsstörung zu beschleunigen, verordnen Sie zudem Prednisolon (70 mg für 2 Wochen, dann in absteigender Dosierung). 6 Wochen nach dem Akutereignis sehen Sie die Patientin erneut in Ihrer Ambulanz. Die Bewegungsstörung hat sich weitgehend zurückgebildet, sodass Sie auch die Medikation mit Tiaprid abdosieren.

Daher sollte die Bewegungsstörung Auslöser für eine Fokussuche sein (Blutkulturen, TEE, Abdomensonografie). Auch weitere Organmanifestationen des rheumatischen Fiebers (Karditis, Arthritis) sollten ausgeschlossen werden.

\section{Therapie}

In der Regel erfolgt auch ohne Nachweis einer persistierenden Infektion (bzw. vor Eingang des Ergebnisses) eine Antibiotikatherapie mit Penicillin G (3 × 3 Mio. IE i. v. ) über 10 Tage, bei Penicillin-Allergie mit Clarithromycin (erster Tag $2 \times 500 \mathrm{mg}$, dann $2 \times 250 \mathrm{mg}$ ). Systemische Entzündungszeichen werden zusätzlich mit NSAID behandelt. In schweren Fällen ist eine immunsuppressive Behandlung mit Prednisolon indiziert. Eine Wirkung intravenöser Immunglobuline ist in der weiteren Eskalation ebenfalls belegt.

Da Reinfektionen mit Streptokokken mit einem schwerer verlaufenden Rezidiv verbunden sein können, wird nach pädiatrischer Leitlinie eine Reinfektionsprophylaxe mit dem Depot-Penicillin Benzathinpenicillin G (1,2 Mega-IE i. m. alle 4 Wochen), alternativ Penicillin V $2 \times 250$ mg/d oder Erythromycin $2 \times 250 \mathrm{mg} / \mathrm{d}$ oral empfohlen, ohne Karditis über 5 Jahre, mit Karditis über 10 Jahre bzw. mindestens bis zum 40. Lebensjahr. Das Risiko für ein Rezidiv nimmt allerdings mit dem Alter ab; für Patienten oberhalb des 40 . Lebensjahrs gibt es daher keine datenbasierten Empfehlungen zur Wertigkeit einer Reinfektionsprophylaxe.

\section{Merke}

Bei Verdacht auf Chorea minor persistierende Infektionen ausschließen bzw. behandeln.

\section{KERNAUSSAGEN}

- Die Huntington-Krankheit beinhaltet motorische, psychiatrische und kognitive Symptome.

- Die motorischen Symptome sollten nur soweit behandelt werden, wie sie den Patienten funktionell einschränken.

- Die Behandlung der psychiatrischen Symptome ist für die Lebensqualität und die Prognose entscheidend.

- Physiotherapie, Ergotherapie, Logopädie und Sozialarbeit sind wichtige Teile der Behandlung. 
- Ein Einbeziehen der Angehörigen ist sinnvoll; sie sind oft gleichzeitig Versorgende und mögliche Genträger.

\section{Wissenschaftlich verantwortlich gemäß Zertifizierungsbestimmungen}

Wissenschaftlich verantwortlich gemäß

Zertifizierungsbestimmungen für diesen Beitrag ist Prof. Dr. med. Björn Falkenburger, Dresden.

\section{Zitierweise für diesen Artikel}

Fortschritte der Neurologie · Psychiatrie 2020; 88(6): 403-415. Dieser Beitrag ist eine aktualisierte Version des Artikels: Falkenburger, B. Huntington-Krankheit und Chorea minor. Neurologie up2date 2019; 2(02): 135-148

\section{Interessenkonflikt}

Der Autor gibt an, dass kein Interessenkonflikt besteht.

\section{Autorinnen / Autoren}

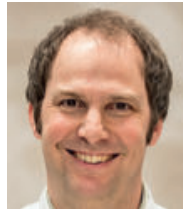

\section{Björn Falkenburger}

Prof. Dr. med. Medizinstudium an der Universität Tübingen. Promotionsarbeit an der Boston University. Facharztweiterbildung in Tübingen, Göttingen und Aachen. Postdoc in Seattle. 2013 Oberarzt, 2014 W1-Professur für translationale Hirnforschung und geschäftsführender Oberarzt der Klinik für Neurologie an der RWTH Aachen. Seit 2019 Professur für Neurologie mit Schwerpunkt Bewegungsstörungen an der TU Dresden.

\section{Korrespondenzadresse}

Univ.-Prof. Dr. med. Björn Falkenburger

Universitätsklinikum Carl Gustav Carusan

der Technischen Universität Dresden

Klinik und Poliklinik für Neurologie

Fetscherstraße 74

01307 Dresden

E-Mail: bjoern.falkenburger@uniklinikum-dresden.de

\section{Literatur}

[1] Oosterloo M, Bijlsma EK, van Kuijk SM et al. Clinical and genetic characteristics of late-onset Huntington's disease. Parkinsonism Relat Disord 2019; 61: 101-105

[2] Honrath P, Dogan I, Wudarczyk O et al. Risk factors of suicidal ideation in Huntington's disease: literature review and data from Enroll-HD. J Neurol 2018; 265: 2548-2561

[3] Aziz NA, van der Burg JMM, Tabrizi S] et al. Overlap between age-at-onset and disease-progression determinants in Huntington disease. Neurology 2018; 90: e2099-e2106
[4] Arrasate M, Mitra S, Schweitzer ES et al. Inclusion body formation reduces levels of mutant huntingtin and the risk of neuronal death. Nature 2004; 431: 805-810

[5] Trajkovic $\mathrm{K}$, Jeong $\mathrm{H}$, Krainc D. Mutant huntingtin is secreted via a late endosomal/lysosomal unconventional secretory pathway. J Neurosci 2017; 37: 9000-9012

[6] McNally G, Rickards H, Horton M et al. Exploring the validity of the short version of the Problem Behaviours Assessment (PBA-s) for Huntington's disease: A Rasch analysis. J Huntingtons Dis 2015; 4: 347-369

[7] Müller HP, Huppertz H], Dreyhaupt ] et al. Combined cerebral atrophy score in Huntington's disease based on atlas-based MRI volumetry: Sample size calculations for clinical trials. Parkinsonism Relat Disord 2019. doi:10.1016/j.parkreldis. 2019.02.004

[8] Saft C, Bonelli RM, Burgunder J-M et al. Leitlinien „Chorea/ Morbus Huntington“ für die deutschsprachigen Länder neu überarbeitet. Akt Neurol 2018; 45: 570-585

[9] Saft C, Hein von SM, Lücke T et al. Cannabinoids for treatment of dystonia in Huntington's Disease. J Huntingtons Dis 2018; 7: 167-173

[10] Fritz NE, Rao AK, Kegelmeyer D et al. Physical therapy and exercise interventions in Huntington's disease: a mixed methods systematic review. J Huntingtons Dis 2017; 6: $217-$ 235

[11] Quinn L, Busse M. Physiotherapy clinical guidelines for Huntington 's disease. Neurodegener Dis Manag 2012; 2: 21-31

[12] Reilmann R, McGarry A, Grachev ID et al.; European Huntington 's Disease Network; Huntington Study Group investigators. Safety and efficacy of pridopidine in patients with Huntington 's disease (PRIDE-HD): a phase 2, randomised, placebocontrolled, multicentre, dose-ranging study. Lancet Neurol 2019; 18: 165-176

[13] Wojtecki L, Groiss S], Ferrea S et al. Surgical Approaches Working Group of the European Huntington's Disease Network (EHDN). A prospective pilot trial for pallidal deep brain stimulation in Huntington's disease. Front Neurol 2015; 6: 180

[14] Surgical Approaches Working Group of the European Huntington 's Disease Network (EHDN); Ferrea S, Groiss SJ, Elben $S$ et al. Pallidal deep brain stimulation in juvenile Huntington's disease: local field potential oscillations and clinical data. J Neurol 2018; 265: 1573-1579

[15] Huntington Study Group. Unified Huntington's Disease Rating Scale: reliability and consistency. Mov Disord 1996; 11: 136142

[16] Dubois B, Slachevsky A, Litvan I et al. The FAB: A frontal assessment battery at bedside. Neurology 2000; 55: 1621 1626

[17] Harbo HF, Finsterer ], Baets ] et al.; EFNS. EFNS quidelines on the molecular diagnosis of neurogenetic disorders: general issues, Huntington's disease, Parkinson's disease and dystonias. Eur J Neurol 2009; 16: 777-785

\section{Bibliografie}

DOI https://doi.org/10.1055/a-1124-0060

Fortschr Neurol Psychiatr 2020; 88: 403-415

(c) Georg Thieme Verlag KG Stuttgart · New York

ISSN 0720-4299 


\section{Punkte sammeln auf CME.thieme.de}

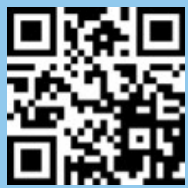

Diese Fortbildungseinheit ist in der Regel 12 Monate online für die Teilnahme verfügbar.

Den genauen Einsendeschluss finden Sie unter https://eref.thieme.de/CXEP1A4.

Sollten Sie Fragen zu Online-Teilnahme haben, finden Sie unter https://cme.thieme.de/hilfe eine ausführliche Anleitung. Wir wünschen viel Erfolg beim Beantworten der Fragen!

Unter https://eref.thieme.de/CXEP1A4 oder über den QR-Code kommen Sie direkt

zur Startseite des Wissenstests.

VNR 2760512020158723664

\section{Frage 1}

Welche der folgenden Aussagen trifft nicht zu:

A Eine Huntington-Krankheit ist auch bei leerer Familienanamnese möglich.

B Wenn die Huntington-Krankheit von der Mutter geerbt wird, ist der Erkrankungsbeginn häufig in einem ähnlichen Alter.

C Wenn die Huntington-Krankheit vom Vater geerbt wird, ist der Erkrankungsbeginn häufig in einem jüngeren Alter.

D Die jugendliche Variante der Huntington-Krankheit ist durch besonders wenige CAG-Wiederholungen gekennzeichnet.

E Bei rasch progredienten Verläufen findet man häufig eine höhere Anzahl an CAG-Wiederholungen.

\section{Frage 2}

Welche der folgenden Aussagen trifft zu:

A Alle Zellen des Körpers haben die gleiche Anzahl an CAGWiederholungen.

B Bei jeder Anzahl an CAG-Wiederholungen kann zweifelsfrei vorhergesagt werden, ob eine Huntington-Krankheit auftreten wird.

C Verhaltensauffälligkeiten vor Beginn der Chorea sind untypisch.

D Suizide sind eher selten.

E Neuroleptika können Symptome einer Huntington-Krankheit mildern.

\section{Frage 3}

Welche der folgenden Aussagen zur Huntington-Krankheit trifft nicht zu?

A Die Bewegungsstörung ist meist beidseitig.

B Choreatische Bewegungen können zumindest kurz unterdrückt werden.

C Die Okulomotorik ist häufig gestört.

D Dystone Bewegungsstörungen sind Teil der Erkrankung.

E Das Fingertippen ist häufig verlangsamt.

\section{Frage 4}

Welche der folgenden Symptome spricht für eine Huntington-Krankheit?

A Hyperkinesien vor allem im Bereich von Mund und Gesicht

B Teleangiektasien der Sklera

C Depression
D Kardiomyopathie

E rasche Progredienz

\section{Frage 5}

Welcher der folgenden Befunde im Schädel-MRT spricht für eine Huntington-Krankheit:

A Atrophie des Nucleus caudatus

B Eisenablagerungen im Striatum

C Verkalkungen im Striatum

D Striatale Hyperintensität in der T1-Wichtung

E Atrophie des Kleinhirns

\section{Frage 6}

Wodurch ist die Substanz Tiaprid gekennzeichnet?

A Tiaprid bewirkt in der Regel eine stärkere Reduktion der Hyperkinesen als Tetrabenazin.

B Tiaprid sollte bis zum Sistieren der Hyperkinesen aufdosiert werden.

C Eine Dosisreduktion im Verlauf der Erkrankung ist meist nicht sinnvoll.

D Bei einer raschen Zunahme der Hyperkinesen sollte die Tiaprid-Dosis gesteigert werden.

E Tiaprid hat ein geringeres Risiko für depressive Nebenwirkungen als Tetrabenazin.

\section{Frage 7}

Welches der folgenden Medikamente verbessert die Hyperkinesen in der Regel nicht?

$\begin{array}{ll}\text { A } & \text { Risperidon } \\ \text { B } & \text { Olanzapin } \\ \text { C } & \text { Clozapin } \\ \text { D } & \text { Aripiprazol } \\ \text { E } & \text { Quetiapin }\end{array}$

\section{Frage 8}

Eines der folgenden Symptome wird durch Amitriptylin nicht behandelt. Welches?

$\begin{array}{ll}\text { A } & \text { Schlafstörung } \\ \text { B } & \text { Hypersalivation } \\ \text { C } & \text { Depression } \\ \text { D } & \text { kognitive Einschränkung } \\ \text { E } & \text { Inkontinenz }\end{array}$

E Inkontinenz 


\section{Punkte sammeln auf CME.thieme.de}

Fortsetzung ...

\section{Frage 9}

Welche Auswahl des Antidepressivums ist nicht sinnvoll?
A Depression und Schlafstörung - Amitriptylin
B Depression und Übergewicht - Mirtazapin
C Depression und Hyperkinesien - Aripiprazol
D Depression und Reizbarkeit-Opipramol
E Depression und Angst-Clonazepam

\section{Frage 10}

Welche Aussage trifft nicht zu?

A Die Wirksamkeit von Physiotherapie ist nicht in Studien untersucht.

B Suizidalität sollte regelmäßig angesprochen werden

C Eine gesetzliche Betreuung ist frühzeitig sinnvoll.

D Die Frage einer PEG-Sonde sollte frühzeitig diskutiert werden.

E Die Frage einer Tracheotomie sollte frühzeitig diskutiert werden. 